\title{
FIXED POINTS OF AUTOMORPHISMS
}

\author{
A. W. CURRIER
}

\begin{abstract}
We prove that the fixed point set of a compact Lie group of automorphisms of a Lie group has finitely many components if the Lie group does.
\end{abstract}

We call a space almost connected if it has only finitely many components. If $H$ is a group acting on a set $S$ let $S^{H}$ be the set of fixed points of $H$. In [2] by $\mathrm{S}$. S. Koh it is shown that if $G$ is an almost connected Lie group and $A$ is a finite automorphism group of $G$, then $G^{A}$, the set of fixed points of $A$, is also almost connected. In this note we prove the following result.

TheOREM. If $G$ is an almost connected Lie group and $A$ is a compact Lie group acting on $G$ as a Lie transformation group of automorphisms, then $G^{A}$ is almost connected.

Proof. As usual we define the semidirect product $\tilde{G}=A \tilde{\times} G$ by $(\alpha, g)$ - $(\beta, h)=\left(\alpha \beta,\left(\beta^{-1} g\right) h\right)$ where $\alpha, \beta$ are in $A$ and $g, h$ are in $G$. Since $A$ is compact, $\tilde{G}$ is an almost connected Lie group.

There is a compact subgroup $K$ of $\tilde{G}$ and subspaces $E_{1}, \ldots, E_{n}$ of $\tilde{G}$ satisfying the following condition: $A \subset K, E_{i}=\exp \mathcal{S}_{i}(i=1, \ldots, n)$, where $\delta_{i}$ is a subspace of $\tilde{\mathfrak{g}}$, the Lie algebra of $\tilde{G}$. Here exp is a diffeomorphism and $\delta_{i}$ is Ad $K$-invariant. Finally, $\tilde{G}=K \cdot E_{1} \cdots E_{n}$ (topologically direct) [ $1, \mathrm{p}$. 180, Theorem 3.1].

$A$ and $G$ are subgroups of $\tilde{G}$ via the identification of $\alpha$ with $(\alpha, e)$ and $g$ with $(I, g)$ where $\alpha$ is in $A$ and $g$ is in $G$. (The identities of $G$ and $A$ are denoted by $e$ and $I$.) We have $\tilde{G}^{A}=K^{A} \cdot E_{1}^{A} \cdots E_{n}^{A}$ where $A$-action on $\tilde{G}$ is conjugation. Since $K$ is compact and each $\varsigma_{i}$ is linear, $\tilde{G}^{A}$ is almost connected.

Now we will show $G^{A}$ is almost connected. Suppose $(\alpha, g)$ is in $\tilde{G}^{A}$. Then $(\beta, e)(\alpha, g)(\beta, e)^{-1}=(\alpha, g)$ for all $\beta$ in $A$. Moreover

$$
\begin{aligned}
(\beta, e)(\alpha, g)(\beta, e)^{-1} & =(\beta, e)(\alpha, e)\left(\beta^{-1}, e\right)(\beta, e)(I, g)\left(\beta^{-1}, e\right) \\
& =\left(\beta \alpha \beta^{-1}, e\right)(I, \beta(g))=\left(\beta \alpha \beta^{-1}, \beta(g)\right)=(\alpha, g) .
\end{aligned}
$$

Hence $\beta \alpha \beta^{-1}=\alpha, \beta(g)=g$ and consequently $(\alpha, g)$ is in $\tilde{G}^{A}$ if and only if $\alpha$ is in $A^{A}$ and $g$ is in $G^{A}$. Hence $G^{A}=\Pi_{2} \tilde{G}^{A}$ where $\Pi_{2}$ is the coordinate projection of $A \tilde{\times} G$ onto $G$.

Therefore $G^{A}$ is almost connected.

Received by the editors November 11, 1974 and, in revised form, December 30, 1974.

AMS (MOS) subject classifications (1970). Primary 54H15, 57E15. 
REMARK. If $A$ is a connected Lie group with simple Lie algebra, then the above result does not hold. Use $A=G=$ the universal covering group of $\mathrm{SL}(2, R)$ and let $A$ act by conjugation.

\section{BIBLIOGRAPHY}

1. G. Hochschild, The structure of Lie groups, Holden-Day, San Francisco, Calif., 1965. MR 34 \#696.

2. S. S. Koh, On affine symmetric spaces, Trans. Amer. Math. Soc. 119 (1965), 291-309. MR $32 \# 1643$.

Department of Mathematics, University of Maryland, College Park, Maryland 20742 